\title{
Software Define Radio Supported Reconfigurable for UMTS Up-Converter
}

\author{
Ibrahim Khider \\ Sudan University of Science \\ and Technology
}

\author{
Ali Ahmed Ibrahim \\ University of Science \\ and Technology
}

\begin{abstract}
Future of wireless communication system designed to merge a variety of services such as voice, data, image and video. These services have various demands on the bandwidth and data rate. So studies has been done to access these requirements, Software Define Radio ( SDR) is being presented as technique offers the possible revolutions the way radios are manufacturing dissemination and used SDR promises to increase flexibility, expand hardware life time and lower costs. In this paper the simulation is being obtained by using MATLAB program for UMTS digital up-convertor to increase data rate as application of SDR.
\end{abstract}

\section{Keywords}

Software Define Radio, Digital Up converter, matlab, Xilinx

\section{INTRODUCTION}

The twentieth century saw the explosion of hardware defined radio (HDR) most radios are hardware defined with little or no software control, they are fixed in function for mostly consumer items for broadcast reception. They have a short life and are designed to be discarded and replaced.[1] Software defined radio (SDR) is an exciting new field for the wireless industry; it is gaining momentum and beginning to be included in commercial and defense products. Software defined radio uses programmable digital devices to perform the signal processing necessary to transmit and receive baseband information at radio frequency. Devices such as digital signal processors (DSPs) and field programmable gate arrays (FPGAs) use software to provide them with the required signal processing functionality. [2] All of the 3G systems are potential SDR applications. Software radio offers the potential to solve many of the problems caused by the proliferation of new air interfaces. Base stations and terminals using SDR architectures can support multiple air interfaces during periods of transition and be easily software upgraded. Intelligent SDRs can detect the local air interface and adapt to suit the need; this capability will be valuable for frequent inter country travelers. [3][4]VHDL stands for VHSIC Hardware Description Language. VHSIC is itself an abbreviation for Very High Speed Integrated Circuits. A fundamental motivation to use VHDL is that VHDL is a standard, technology/vendor independent language, and is therefore portable and reusable. VHDL is a hardware description language. It describes the behavior of an electronic circuit or system. The two main immediate applications of VHDL are in the field of Programmable Logic Devices (including CPLDs - Complex Programmable Logic Devices and FPGAs-Field Programmable Gate Arrays) and in the field of ASICs (Application Specific Integrated Circuits) [1]. A final note regarding VHDL is that, contrary to regular computer programs which are sequential, its statements are inherently concurrent (parallel). For that reason, VHDL is usually referred to as a code rather than a program. In VHDL, only statements placed inside a PROCESS, FUNCTION, or PROCEDURE are executed sequentially. [1] Xilinx ISE is a comprehensive synthesis and implementation environment for Xilinx programmable devices. ModelSim XE (from Model Technology) is also provided as part of the package. The former is employed for circuit synthesis and design implementation, while the latter is used for simulation. Most digital filter designs are either infinite impulse response (IIR) type or finite impulse response (FIR) type. Digital FIR filters are linear systems and because they process sampled Data they are also discrete in time. The major advantage of the FIR filter is that it is possible to achieve an exact linear phase response with unconditionally stability. For this reason it is the most popular choice of filter for use in digital communications.

\section{SYSTEM MODEL EPRESENTATION}

The figure below shows the elements of model based Design. The center focus of this design methodology is a model, whose four Main elements are: Executable specifications, Design with simulation Implementation with code generation, Continuous test and verification. Model-based design helps to create better embedded software and hardware by increasing the accuracy and speed of system Development. We can confidently begin integration, test, and deployment of our embedded application knowing that we have identified design errors and met our requirements. Model-based design provides a proven solution that reduces development time and cost and fosters quality and innovation in the development of embedded systems. [5][6] To calculate the output sample rate $(\mathrm{Fs})$ we use the figure .2 to easy calculate. The interpolation filter means multiplication the input signal by two. Mathematical representations: The calculations of interpolations filter summarized in table 1 . 


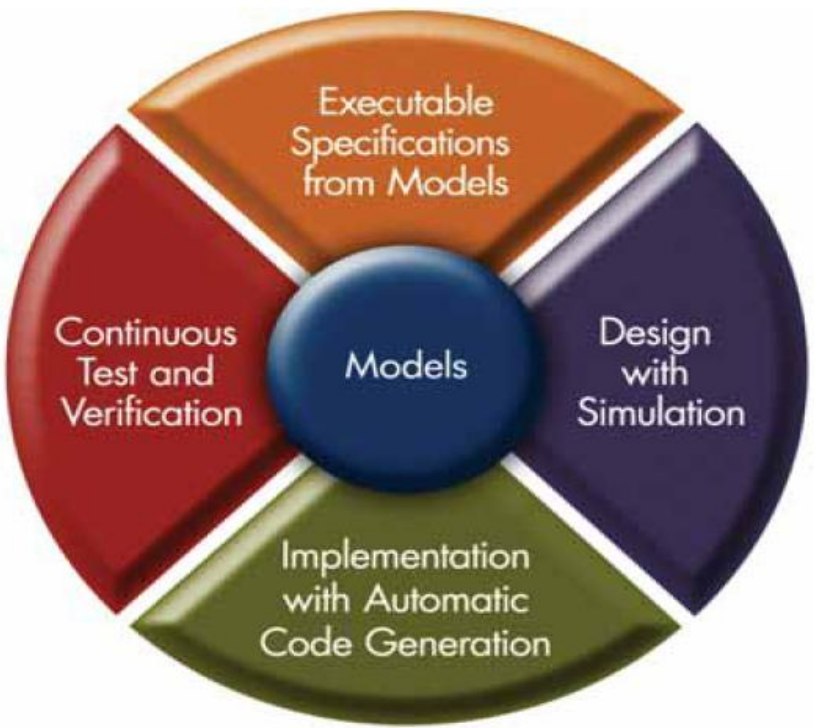

Figure 1: Elements of Model Based Design

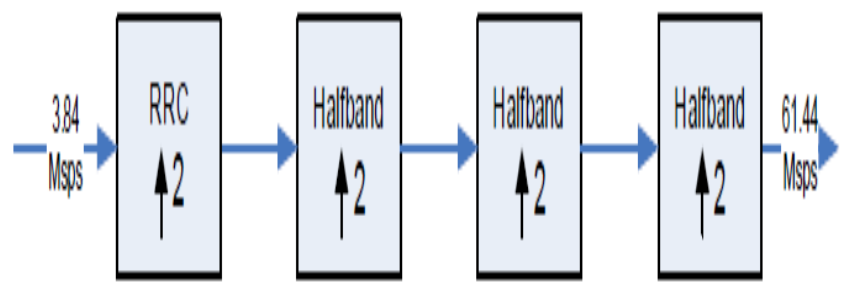

Figure2: DUC interpolation Filter

Table 1: Interpolation Filters

\begin{tabular}{|l|c|c|c|c|}
\hline \multicolumn{1}{|c|}{ Filter Stage } & $\begin{array}{c}\text { Passband } \\
\text { Fpass (MHz) }\end{array}$ & $\begin{array}{c}\text { Output Sample Rate } \\
\text { Fs (MSPS) }\end{array}$ & $\begin{array}{c}\text { Peak-10.Peak } \\
\text { Ripple (dB) }\end{array}$ & Filter Order \\
\hline Windowed RRC & 1.92 & 7.68 & 0.012 & 44 \\
\hline First Halltband & 2.34 & 15.36 & 0.002 & 22 \\
\hline Second Halthand & 2.34 & 30.72 & 0.002 & 10 \\
\hline Third Hallband & 2.34 & 61.44 & 0.001 & 10 \\
\hline
\end{tabular}

\section{SIMULATION PART}

We use matlab to simulate our model which provides the pulse shaping, interpolation, and frequency translation to the singlecarrier baseband WCDMA signal from $0 \mathrm{~Hz}$ to a set of specified center frequencies. It is designed to meet the 3rd Generation Partnership Project (3GPP) specification, which defines the transmission and reception requirements for the base station radio. [3]

DUC Filter Design: a)

When it comes to filter architectural consideration, both the system performance and the hardware resource usage should be taken into account. The interpolation filter chain in the DUC needs to pulseshape and up sample the baseband data by a factor of $61.44 / 3.84=16$.

b) First, the filters should be designed in each configuration to obtain the filter length. This is produced by using the MATLABC Filter Design and Analysis Tool (FDATool).

c) The FDATool has a graphical user interface (GUI) that allows users to set filter specifications

From Figure2 it shows that the parameters of design digital filter which are: Response type, Design method, Filter order, Frequency specifications and Magnitude specifications.

Design of RRC Filter:

To design RRC filter we use the graphical user interface of FADTOOL with this specifications: In Response type use Raised-cosine because has no inter-chip interference (ICI) and the zero crossings occur at chip intervals. However, it has an infinite response in the time domain. In order to determine design method a common method is to use the window approach to design a filter within a manageable length. And use FIR -Window for Symmetric Tap. Filter order (44) to achieve similar performance than various windows. Option we chose Chebyshev - Window to provide better side lobe suppression than a rectangular window at the expense of some widening of the main lobe. We select Frequency specifications in $\mathrm{MHz}$, select the value of $\mathrm{Fs}=7$. 68, because the RRC filter use as interpolation in DUC therefore the chiprate complex input multiply by 2 ( $\mathrm{fs}=3.84 * 2=7.68) \&$ Roll off $=0.22$ Magnitude specification is Square root. This parameter which is inserted in FDATOOL gives the output in figure 2.figure 3 illustrate response of $\mathrm{RRC}$ filter represented the relationship between frequency in $\mathrm{MHz}$ (x-axis) and magnitude in $\mathrm{dB}$ ( $\mathrm{Y}$-axis). from $0-2.5 \mathrm{MHz}$ passes band and from $2.5 \mathrm{MHz}$ stop-band or side-lope .

First Half band Interpolator:

Half band filters are a type of FIR filter where its transition region is centered at one quarter of the sampling rate, Fs/4. The output sample rate of the filter is four times the chip rate, that is, Fs $=3.84 * 4=15.36 \mathrm{MSPS}$. The pass band was set to Fpass $=3.84 * 1.22 / 2=2.34 \mathrm{MHz}$ and the pass band ripple is chosen to be $0.002 \mathrm{~dB}$. Using the MATLAB FDATool. The frequency (magnitude) response of the filter is shown in figure 4 . figure 4 illustrate response of First half band filter represented the relationship between frequency in $\mathrm{MHz}$ (x-axis) and magnitude in $\mathrm{dB}$ (Y-axis).we observe that increase pass band and decrease the magnitude of side-lope.

Second Half band Interpolator:

After the first interpolation filter, the signal is again up sampled by a factor of two using a half band interpolator. The output sample rate of the filter is eight times the chip rate, that is, Fs $=3.84 * 8=30.72$ MSPS. The pass band is the same as the first interpolator, set to Fpass $=2.34 \mathrm{MHz}$ The pass band ripple was chosen to be $0.002 \mathrm{~dB}$. Half band filter was designed, and its magnitude response is shown in figure 5 . The figure 6 illustrate response of Second half band filter we observe that increase pass band and decrease the magnitude of side-lope.

Third Half band Interpolator:

Finally, the signal enters the last half band interpolator to reach an output sample rate of $61.44 \mathrm{MSPS}$. The pass band was set to Fpass $=3.84 * 1.22 / 2=2.34 \mathrm{MHz}$, identical to the first two interpolators. The pass band ripple was chosen to be $0.001 \mathrm{~dB}$. 
half band filter was designed to this specification with its magnitude response shown in figure 6.figure 6 illustrates response of Third half band filter there is no side-lope and all signal is pass band.

\section{RESULTS AND DISCUSSION}

Figure8 shows that Relationship between frequency in $\mathrm{MHz}$ (x-axis) and magnitude in $\mathrm{dB}$ (Y-axis). In the model filter design we specify the parameters which get this shape we observe that the signal main lope has magnitude around zero position only because FIR filter it is use impulse signal. figure 9 shows that the final output signals after passes to multiplier and additions. The multiplier used as shifted the signal coming from interpolator filter to certain value (shifted from around zero position to certain value assume around $15 \mathrm{MHz}$ position) delay or advance. 


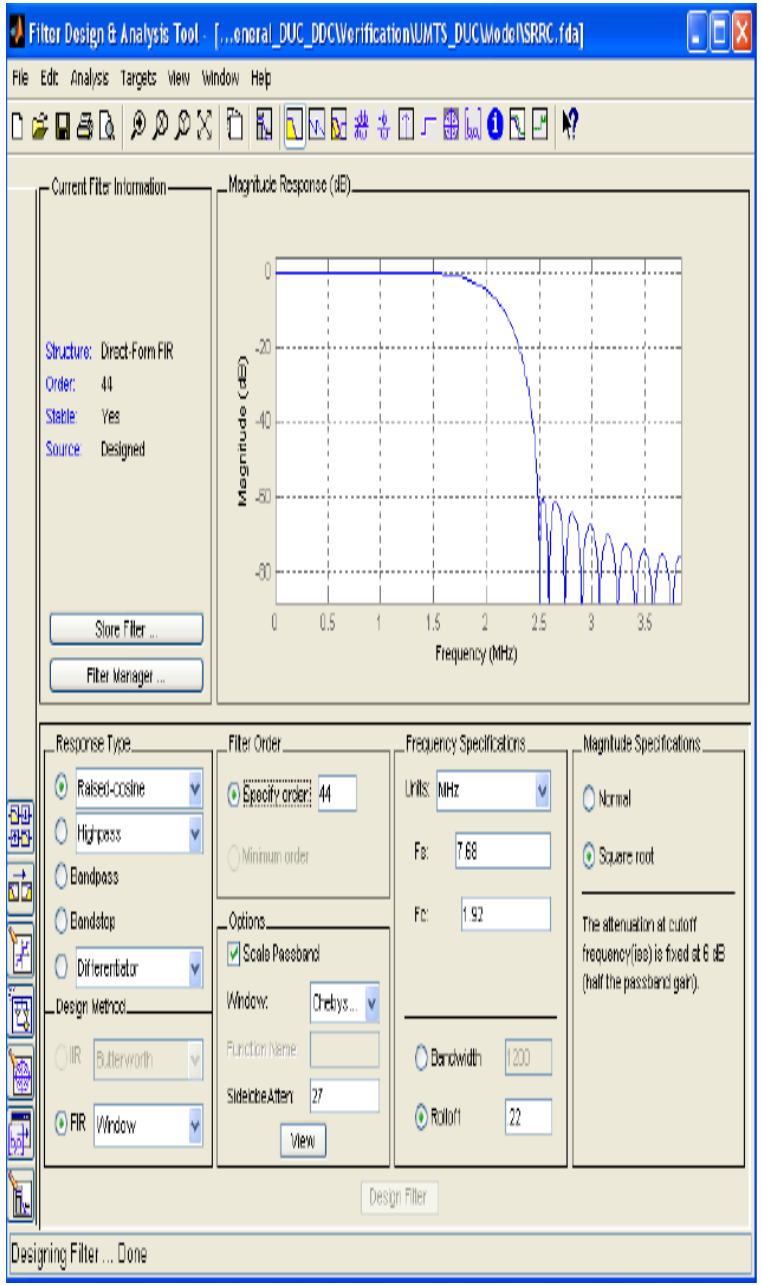

Figure 2: FDATOOL for Design Digital Filter

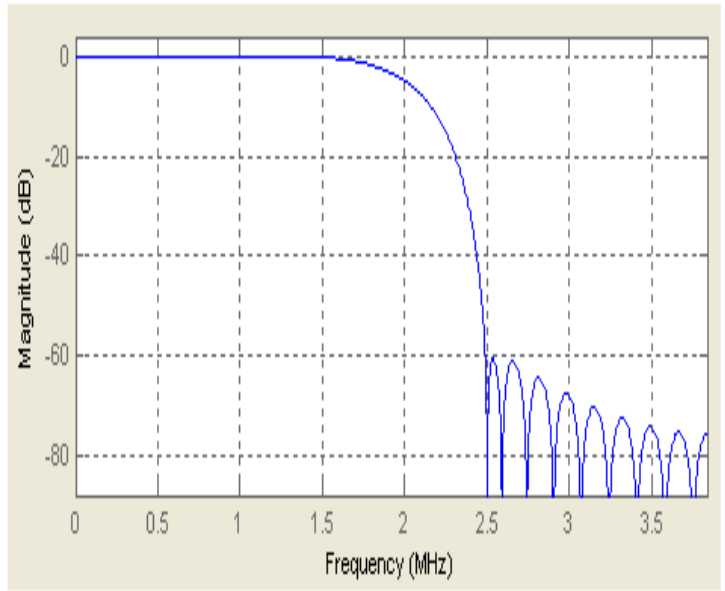

Figure 3: Magnitude Response of RRC Filter 


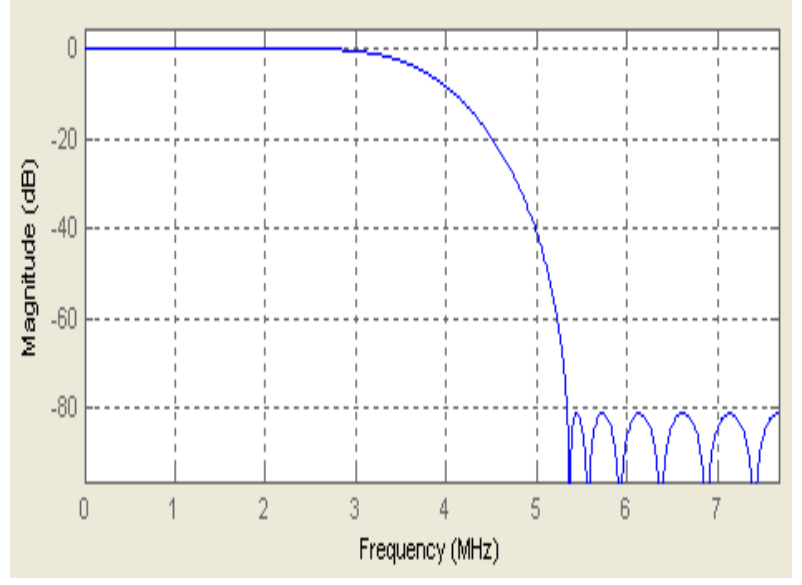

Figure4. Magnitude Response of First Interpolator Filter

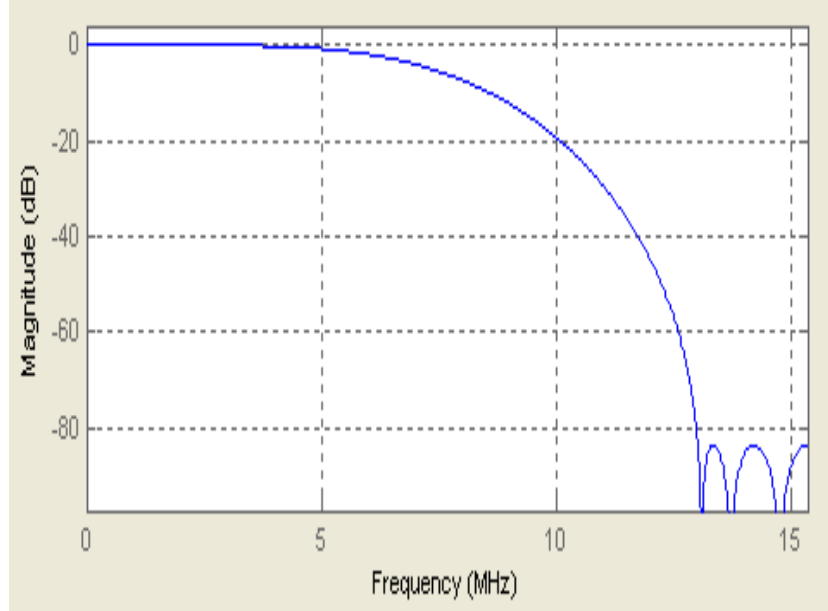

Figure 5: Magnitude Response of Second Interpolator Filter

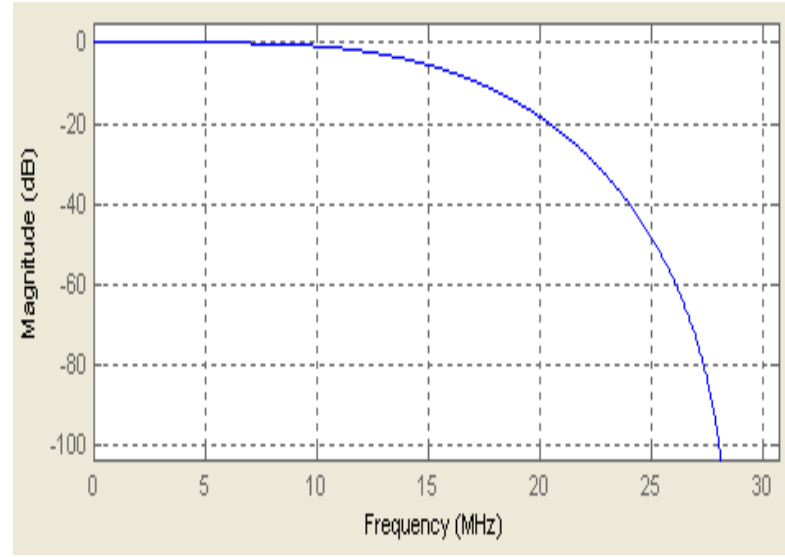

Figure 6: Magnitude Response of Third Interpolator Filter 


\section{CONCLUSION}

This paper has concentrated on SDR and aim to improve performance of the software and hardware implementation sensitivity and increase the flexibility to reduce the design errors. Also SDR has more challenge when we use FPGA technology that offer and provide more efficiency to process the signal.It was seen clearly that using SDR technique to improves the performance of the system and overcome the previous challenges. Yet there are several challenges that inhibit the progress of SDR and Researchers throughout the world are contributing their ideas to solve these challenges. SDR is very sensitive when implemented programming the required device. it is better to implement SDR more accurate to avoid the complexity which faced the designer .

\section{REFERENCES}

[1] Paul Burns,2007, "Software Define Radio", Springer Science Business Media, LLCNY 10013, USA.

[2] Claus, C., Zhang, B., Stechele, W., Braun, L., H“ubner, M., Becker, J.: A ,2008, multi platform controller allowing for maximum dynamic partial reconfiguration through- put. In: Field Programmable Logic and Applications, pp. 535-538. IEEE, Los Alamitos

[3] Uhm, M.:,2005,Software-defined radio: The new architectural paradigm. Xilinx DSP Magazin, 40-42 .

[4] Xilinx Inc. Virtex-5 Family ,2007, Platfrom Overview LX and LXT Platforms v2.2 Tessier, R., Swaminathan, S., Ramaswamy, R., Goeckel, D., Burleson, W.:2005, A re-configurable, power-efficient adaptive Viterbi decoder. IEEE Transactions on VLSI Systems 13(4), 484-488

[5] WWW.Xilinx.com Accessed MAY 2012

[6] M. Stylianou, J. Antoniou, A. Pitsillides, G. Hadjipollas, and V. Vassiliou 2004,"Interfacing Issues for a system level simulator for EUMTS," in Proc. SEACORN Workshop on Simulation of Enhanced UMTS Access and Core Networks,Cambridge, UK. 